\title{
Comunicación
}

\section{Litiasis renal y vesical en un canino: descripción imagenológica}

\author{
Kidney and bladder lithiasis in a canine: imaging description
}

Renso Gallego ${ }^{1,3}$, Vanessa Arenas ${ }^{2}$, José Ortiz ${ }^{2}$

\section{Resumen}

Se reporta un caso de urolitiasis en un canino macho Yorkshire de seis años que ingresa a la Clínica Veterinaria de la Corporación Universitaria Lasallista con historia de orina con sangre, dificultad para orinar $y$, a veces, pequeñas cantidades de orina. Se realizaron exámenes complementarios como radiografía simple y contrastada, ecografía abdominal, citoquímico de orina, urocultivo y hemograma con química sanguínea. Los métodos diagnósticos más sensibles fueron la radiografía simple y con medio de contraste que mostraron cuatro estructuras radiopacas en la vejiga urinaria, y la ecografía abdominal donde se pudo observar las paredes vesicales engrosadas y estructuras hiperecoicas de bordes homogéneos compatibles con urolitos. Se realizó la cistotomía para la remoción de los urolitos.

Palabras clave: canino; ecografía abdominal; neumocistografía; radiografía de abdomen; urolitiasis

\section{AbStract}

A case of urolithiasis is reported in a six-year-old Yorkshire male brought to the Veterinary Clinic of the Lasallian University Corporation with a history of bloody urine, difficulties in urinating and, sometimes, small amounts of urine. Complementary tests were performed including simple and contrasted radiography, abdominal ultrasound, urine cytochemistry, urine culture and blood chemistry. The most sensitive diagnostic

\footnotetext{
${ }^{1}$ Corporación Universitaria Remington, Medellín, Colombia

${ }^{2}$ Corporación Universitaria Lasallista, Medellín, Colombia

${ }^{3}$ E-mail: renso.gallego@uniremington.edu.co
}

Recibido: 15 de junio de 2018

Aceptado para publicación: 15 de noviembre de 2018 
methods were simple radiography and contrast media that showed four radiopaque structures in the urinary bladder, and abdominal ultrasound where thickened vesical walls and hyperechoic structures with homogeneous edges compatible with uroliths could be observed. Cystotomy was performed for the removal of uroliths.

Key words: canine; abdominal ultrasound; pneumocystography; abdominal x-ray; urolithiasis

\section{INTRODUCCIÓN}

En la evaluación radiográfica vesical se incluyen como parámetros normales la densidad de fluidos de aspecto homogéneo y el órgano de forma y tamaño variable, ubicado parcialmente en el canal pélvico (Hecht, 2015). La exploración radiográfica con contraste se utiliza para la evaluación diagnóstica del sistema urinario en perros (Temizoglu et al. 2006), la cual implica la administración de agentes de contraste positivos y negativos en la vejiga urinaria (Bartges y Callens, 2015). La cistografía con contraste negativo usando un agente gaseoso como el dióxido de carbono facilita la evaluación vesical, permitiendo la identificación de masas, defectos del llenado luminal, hernias vesicales y urolitos, entre otros (Silverman, 2000). La técnica con contraste positivo permite el diagnóstico de ruptura vesical o la comunicación anormal con estructuras adyacentes (fistula uretrorectal) y hernias abdominales, entre otros (Bischoff, 2003). La cistografía con doble contraste implica la administración de un medio de contraste positivo y uno negativo en la vejiga urinaria para la evaluación de las lesiones de la mucosa vesical y su lumen, la cual se realiza en los casos que no se puede hacer una ecografía (Hecht, 2015).

La técnica ecográfica se realiza con el fin de detectar urolitos, así como el tamaño, forma y densidad vesical (Langston et al., 2008; Bartges y Callens, 2015). Uno de los puntos de evaluación ecográfica es la mucosa vesical, la cual debe ser inferior a $2 \mathrm{~mm}$ de grosor en perros normales (Guillén et al.,
2011). La orina debe tener aspecto anecogénico, donde cambios en su ecogenicidad pueden relacionarse con proteínas, celularidad, acúmulo de cristales y urolitos (Hecht, 2014). Estructuras como los urolitos provocan la presencia de artefactos en la ecografía vesical (Barrett, 2011). La sombra acústica se produce generalmente en presencia de estructuras de composición mineral alojadas en la mucosa de la vejiga, observándose sombras hipo o anecogénicas que se disponen hacia distal de la imagen (Sánchez et al., 2016).

\section{Reporte de CAso}

En abril de 2018 ingresó a la Clínica Veterinaria de la Corporación Universitaria Lasallista, Colombia, un canino macho de raza Yorkshire Terrier de 6 años y $3.5 \mathrm{~kg}$ de peso. El motivo de consulta médica fue de hematuria, disuria y, en algunas ocasiones, oliguria. En el examen clínico a la palpación abdominal se evidenció abdomen tenso con leve molestia y dolor a nivel del mesogastrio mostrando una postura de xifosis.

Se realizaron exámenes complementarios: cuadro hemático, química sanguínea, citoquímico de orina, cultivo y antibiograma de orina, estudio radiográfico abdominal, estudio radiográfico con medio de contraste (Figura 1) y ecografía abdominal (Figura 2).

El examen hematológico mostró una leucocitosis ligera representada por neutrofilia. En el resultado del citoquímico de 


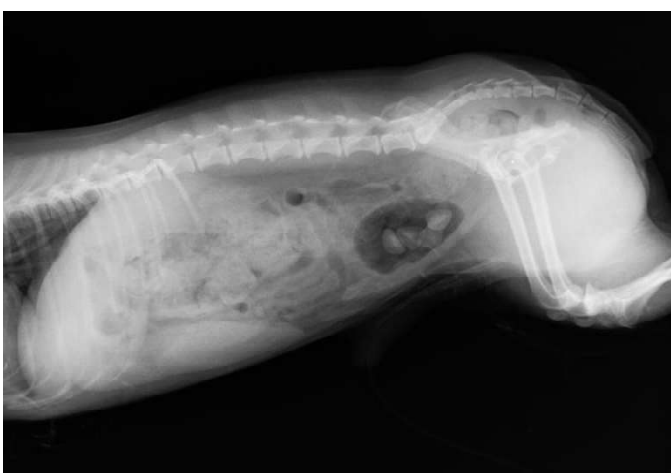

Figura 1. Estudio lateral de abdomen en un perro Yorkshire Terrier con medio de contraste negativo (neumocistografia). Se observan cuatro estructuras radiopacas en el interior de la silueta vesical que se encuentra dilatada por el medio de contraste (aire). orina se evidenció abundante proteinuria, confirmado con una relación albumina-creatinuria en orina de $6 \mathrm{mg} / \mathrm{dl}$. La densidad urinaria era de $1.020 \mathrm{mg} / \mathrm{dl}$ por debajo del rango normal (1.025-1.045 mg/dL). Además, hubo aumento del $\mathrm{pH}(7.2)$, relacionado con la presencia de bacterias ureasas positivas (Staphylococcus pseudintermedius: $>10000 \mathrm{UFC/g}$ ).

Según los signos clínicos durante la anamnesis y los hallazgos de los exámenes clínico, radiográfico y ecográfico de abdomen se emite un diagnóstico diferencial de litiasis renal unilateral (riñón izquierdo) y urolitiasis vesical. Se opta por un tratamiento quirúrgico realizado mediante la técnica de cistoto-mía.
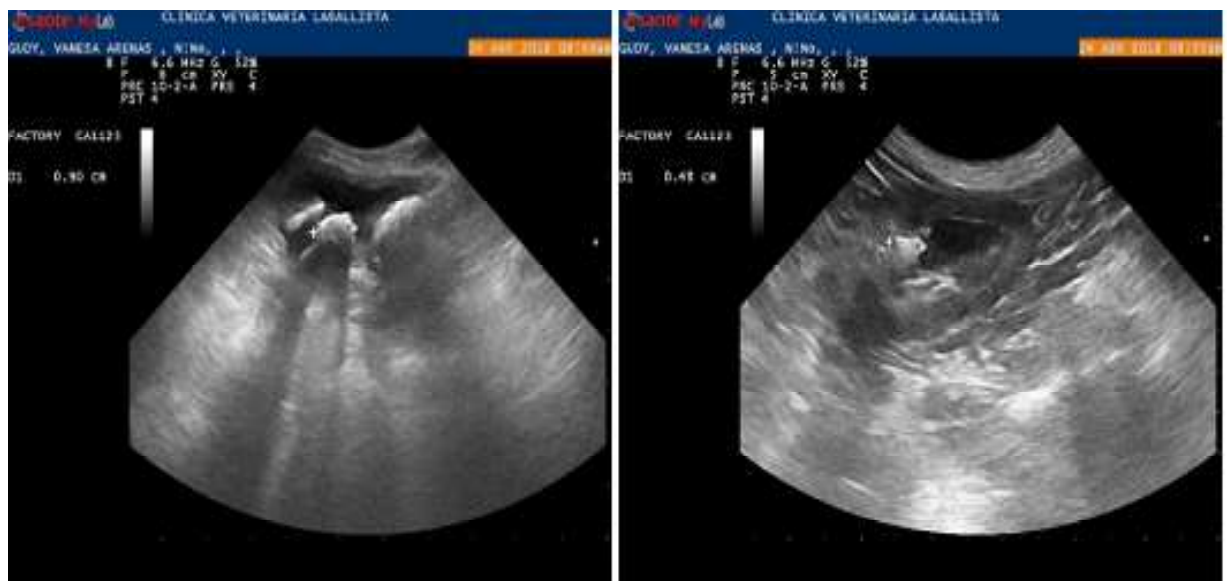

Figura 2. Izquierda. Corte longitudinal de la vejiga. Se evidencian múltiples estructuras hiperecogénicas con presencia de sombra acústica con un diámetro de $0.9 \mathrm{~cm}$ compatible con urolitos. Derecha. Corte longitudinal del riñón izquierdo. Se evidencia una estructura hiperecogénica con sombra acústica $(0.48 \mathrm{~cm})$ a nivel de la pelvis renal. No se mantiene una relación cortico-medular, evidenciándose un aumento en el tamaño en la pelvis renal

En el abordaje quirúrgico de la vejiga se extraen cuatro urolitos (mixtos de oxalato de calcio y de estruvita) (Figura 3). Se realizó el lavado de la vejiga por medio de hidropropulsión interna con el fin de eliminar el sedimento y cristales presentes en vejiga y uretra y se realizó la prueba de permeabilidad.

\section{Discusión}

Freitas (2004) encontró que la evaluación y la cuantificación de urolitos mediante radiografía fue menos sensible cuando estos tenían un tamaño menor de $0.5 \mathrm{~cm}$ o su cantidad era mayor a 20 . Por otro lado, la 


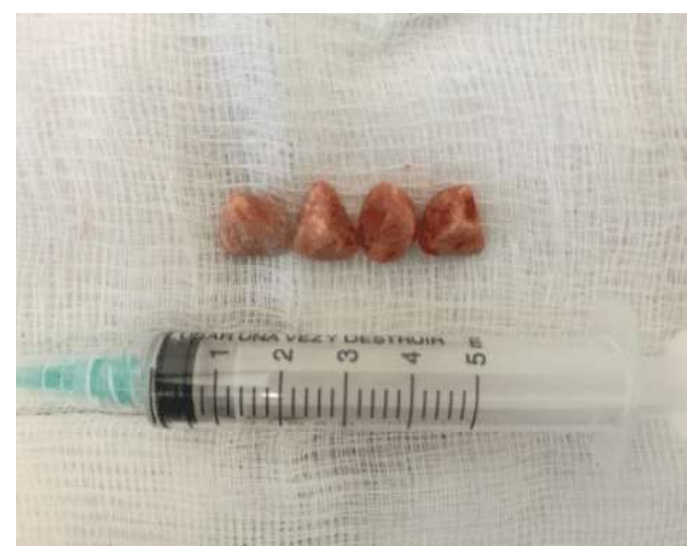

Figura 3. Urolitos vesicales de $0.9 \mathrm{~cm}$ de diámetro en promedio en un perro Yorkshire Terrier.

ultrasonografía vesical aumenta la sensibilidad de su detección (De Lima et al., 2017). En el presente caso se realizó el diagnóstico mediante radiografía contrastada, ya que la radiografía convencional no permitía la evaluación de lo urolitos radiolúcidos; asimismo, por medio de la ultrasonografía transabdominal se hizo la medición de los urolitos vesicales $(0.9 \mathrm{~cm})$ y de los nefrolitos $(0.48 \mathrm{~cm})$.

El procedimiento empleado está de acuerdo con Gallatti e Iwasaki (2004), quienes mencionan que la evaluación y medición de los cálculos radiolúcidos es más sensible cuando se utiliza radiografía de doble contraste. A su vez, indican que el ultrasonido permitió el diagnóstico del $33.3 \%$ de los pacientes, pero que no demostró ser sensible en la cuantificación y medición de los urolitos. Así mismo, De Lima et al. (2017) concluyen que la técnica radiográfica convencional y el ultrasonido tienen la misma sensibilidad en el diagnóstico de urolitos radiopacos y proponen la radiografía de doble contraste como método de elección para la detección de urolitos radiolúcidos.

La sensibilidad de la radiografía como técnica diagnóstica en la evaluación de pacientes con urolitos vesicales se encuentra afectada por la presencia de contenido gastrointestinal, artefactos del tejido óseo y posicionamiento incorrecto del paciente, entre otros (Freitas et al., 2004). La radiografía y la ultrasonografía cobran importancia en el abordaje diagnóstico de la litiasis vesical en caninos (Rosas Martínez et al., 2017). Los procedimientos de estudio radiográfico con medio de contraste y la ultrasonografía aportan información diagnóstica a las patologías vesicales en perros (Feeney, 2003).

A nivel vesical se suelen encontrar urolitos de oxalato de calcio, los cuales presentan una densidad radiopaca en la radiografía (Guillén et al., 2011; Sánchez et al., 2016). A su vez, Syme (2012) señalan que este tipo de urolitos son más prevalentes en caninos y que su ecogenicidad es mayor en comparación a los urolitos de estruvita.

\section{Literatura Citada}

1. Barrett E. 2011. Bladder and urethra. In: BSVA Manual of canine and feline ultrasonography. UK: BSVA. p 155-164.

2. Bartges JW, Callens AJ. 2015. Urolithiasis. Vet Clin N Am-Small 45: 747-768. doi: 10.1016/j.cvsm.2015.03.001

3. Bischoff MG. 2003. Radiographic techniques and interpretation of the acute abdomen. Clin Tech Small An P 1: 7-19. doi : 10.1016/1096-2867(03)90021-1

4. De Lima C, Cintra C, Meirelles AÉ, Crivellenti S, Mariani O, Honsho D, Santana A, et al. 2017. Sensitivity of urolithiasis detection using urinary, radiography and ultrasound parameters. Semin-Cienc Agrar 38: 3599-3604. doi: 10.5433/1679-0359.2017v38n6p3599

5. Feeney $\boldsymbol{D A}$. 2003. The kidney and ureters. In: Veterinary diagnostic radiology. Kindle. p 556-570.

6. Freitas RM, Silva L, Santos JL, Tavares $W$. 2004. Evaluation of imaging methods in the diagnosis of urolithiasis: review of the literature. Radiol Bras 4: 291-294. doi: 10.1590/S0100-39842004000400014 
7. Gallatti LB, Iwasaki M. 2004. Estudo comparativo entre as técnicas de ultrassonografia e cistografia positiva para detecção de alterações vesicais em cães. Braz J Vet Res Anim Sci 41: 40-46. doi: 10.1590/S1413-95962004000-100007

8. Guillén R, Ruíz I, Vera-Vigo P, Ozuna R. 2011. Litiasis recidivante en cachorro Schnauzer miniatura. Reporte de un caso. Mem Inst Investig Cienc Salud 9: 49-53

9. HechtS. 2014. Applications of ultras-ound in diagnosis and management of urinary disease. In: Kirk's Current Veterinary Therapy. USA: Elsevier. p 840-845.

10. Hecht S. 2015. Diagnostic imaging of lower urinary tract disease. Vet Clin N Am-Small 45: 639-663. doi: 10.1016/ j.cvsm.2015.02.002

11. Langston C, Gisselman K, Palma D, McCue J. 2008. Diagnosis of urolithiasis. Compendium 30: 447-450.

12. Rosas A, García LM. 2017. Reporte de urolitiasis vesical en un canino en la clínica veterinaria unipaz. Rev CITECSA 8: 59-69.
13. Sanchez IC, Zea P, Alvarez TM, Monge JF, Parra KL. 2016. Urolitiasis vesical en un canino French Poodle del municipio de Florencia, Caquetá - Colombia: descripción de un caso clínico. REDVET 17(11). [Internet]. Disponible en: http://www.redalyc.org/pdf/636/ 63649051026.pdf

14. Silverman S, Long CD. 2000. The diagnosis of urinary incontinence and abnormal urination in dogs and cats. Vet Clin N Am-Small 2: 427-448. doi: 10.1016/S0195-5616(00)50029-1

15. Syme HM. 2012. Stones in cats and dogs: what can be learnt from them? Arab J Urol 10: 230-239. doi: 10.1016/ j.aju.2012.06.006

16. Temizoylu MD, Bumin A, Kaya M, Alkan Z. 2006. Radiographic and ultrasonographic evaluation of the upper urinary tract diseases in dogs: 22 cases. Ankara Univ Vet Fak 53: 5-13. doi: 10.1501/Vetfak_0000000062 